\title{
Is B-mode ultrasound alone a sufficient screening tool for carotid stenosis? A pilot study
}

Tina Bhandari ${ }^{1}$ and Steven J Socransky ${ }^{2^{*}}$

\begin{abstract}
Background: Carotid ultrasound is performed solely in hospital ultrasound departments or outpatient labs, using both B- and Doppler modes. We hypothesize that B-mode without Doppler can be used to classify patients as having carotid stenosis (CS) above or below 50\%. Our objective is to determine the frequency with which a CS $>50 \%$ is found using Doppler when no such stenosis was visible using B-mode.

Methods: This was a retrospective study of 100 patients referred to the stroke clinic and 100 patients referred for carotid endarterectomy (CEA). All patients had an elective carotid ultrasound done at Health Sciences North. The ultrasound reports were mixed together and blinded. Investigators determined if there was a CS of greater or less than $50 \%$ based on the carotid diagram. These results were compared to the degree of CS found on Doppler.
\end{abstract}

Results: In the CEA group, there were 198 ultrasounds, with 153 showing a CS of $>50 \%$. Only one case of CS $>50 \%$ was missed by B-mode. In the clinic group, 32 of 192 ultrasounds showed a CS of $>50 \%$. None were missed by B-mode. B-mode had a sensitivity and negative predictive value of $100 \%$ and a specificity of $65 \%$.

Conclusion: This study supports the theory that it may be possible to use B-mode ultrasound without Doppler to reliably determine if there is CS above or below 50\%. Further research is required before carotid ultrasound using B-mode alone can be recommended.

Keywords: Carotid stenosis; Ultrasonography; Doppler ultrasonography; Duplex ultrasonography; Point-of-care ultrasonography

\section{Background}

In 2000, cerebrovascular accidents (CVAs) accounted for $7 \%$ of all deaths in Canada [1]. The incidence of CVAs has increased more than $100 \%$ in low to middle income countries in the last four decades, reaching an epidemic level [2].

The main surgical intervention to prevent CVAs is carotid endarterectomy (CEA), which treats stenosis at the level of the carotid bifurcation. CEA decreases the risk of future CVA in both symptomatic and asymptomatic patients [3-5].

Carotid stenosis is usually diagnosed with ultrasound. Elective carotid ultrasound is performed solely by ultrasound technologists and imaging specialists (e.g. radiologists and vascular surgeons). Carotid ultrasound is generally performed on symptomatic patients who present

\footnotetext{
* Correspondence: ssocransky@sympatico.ca

${ }^{2}$ Emergency Department, Health Sciences North, 41 Ramsey Lake Road, Sudbury, ON P3E 5J1, Canada

Full list of author information is available at the end of the article
}

with a possible CVA or a transient ischemic attack. Carotid ultrasound can also be used to screen asymptomatic patients [6].

The yield of screening asymptomatic patients depends on the patient group that is screened. In the general population over 65 years of age, the prevalence of clinically important carotid stenosis is $1 \%$ [7]. In a study of patients who were referred to a vascular surgeon for non-carotid disease, carotid ultrasound found significant stenoses in $21 \%$ of patients [8]. If screening was restricted to patients with a carotid bruit, at least half of significant carotid stenoses would be missed [9]. Risk factors (e.g. hypertension, smoking, cardiac disease, dyslipidemia, or a family history of stroke) place patients at an increased risk of cerebrovascular disease. However, significant stenosis may be found in as little as $1 \%$ of such populations [7]. Screening of patients at risk with elective ultrasound may therefore not be cost-effective.

\section{它}


In many ultrasound labs, elective carotid ultrasound involves the evaluation of the common carotid artery (CCA), the CCA bifurcation, the internal carotid artery (ICA), the external carotid artery (ECA), the vertebral artery, and the subclavian artery. It is performed using both B-mode and Doppler ultrasound, a combination termed 'duplex' ultrasound. This extensive evaluation is time-consuming, requiring $30 \mathrm{~min}$ or more to complete. Most carotid duplex exams are normal or show only mild disease.

When screening for carotid stenosis, only those areas treated by CEA may need to be evaluated. When B-mode shows no evidence of stenosis, evaluation by Doppler may be unnecessary because velocity measurements are normal when the stenosis is less than $50 \%$. Stenoses less than $50 \%$ are not clinically significant and no further investigation would be needed. The use of only B-mode at the level of the carotid bifurcation would make the exam much less time-consuming and possibly tip the cost-benefit ratio in favor of more screening. It also opens up the possibility that clinicians who perform point-of-care ultrasound (POCUS) could add carotid screening to their practice. Only those patients with a screening B-mode ultrasound suggesting a stenosis over $50 \%$ would need to undergo an elective duplex scan for the purposes of measuring velocities.

The primary objective of this study was to determine the frequency with which a carotid stenosis of greater than $50 \%$ is found using spectral Doppler when no such stenosis was visible using B-mode.

\section{Methods}

\section{Study setting and population}

This study took place at Health Sciences North (HSN) in Sudbury, Ontario. HSN is the vascular surgery referral hospital for Northeastern Ontario as well as the Stroke Centre for Sudbury-Manitoulin district. The study was approved by the HSN research ethics committee.

\section{Study design and protocol}

This was a retrospective chart review with blinded data abstraction. A total of 200 patients were included in the study, with 100 patients coming from each of the following groups:

a. One hundred patients were sampled from among those patients who had a pre-operative carotid ultrasound and subsequent CEA performed at HSN.

b. One hundred patients were sampled from among those who were referred to the HSN stroke program and had a carotid ultrasound performed at HSN in association with the referral.

All patients in group a had evidence of significant carotid stenosis on their elective ultrasound on at least one side. Only a minority of scans (16.7\%) performed on patients in group b showed a carotid stenosis greater than $50 \%$. Group b was added to mitigate against bias in data interpretation by the investigators by providing a more appropriate mix of patients with milder degrees of stenoses or normal findings on their ultrasound. Consecutive patients were selected for each group, starting with those patients who had an ultrasound performed in March 2011. Patients were selected going backwards in time until 100 patients were selected for each group.

Once the patients were selected, the ultrasound reports of all patients in both groups were printed and randomly mixed together so that their original group was unidentifiable. Each report was then randomly assigned a number from 1-200 (Figure 1a). Clerical staff then blinded all personal information, flow measurements, and interpretations. The only visible part of the report was the number used to identify the patient and the diagram of the carotid vessels with stenosis, if any, drawn by the ultrasound technologist who conducted the initial exam (Figure 1b).

One study investigator (TB) reviewed all reports (as depicted in Figure $1 \mathrm{~b}$ ) to determine if there was a stenosis of greater than $50 \%$ in each vessel (CCA, ICA, ECA) based on the diagram alone. This was done for both the right and left carotid systems.

A second study investigator (SS) made the same assessment on every fifth report (20\% of the reports) in order to determine inter-rater reliability. This second reviewer was blinded to the data collected by the first reviewer. The results of the two study investigators were then compared and a kappa statistic was calculated.

Following these assessments, the ultrasound reports were unblinded to reveal the velocity measurements and interpretations of stenosis. These findings were compared to the results from the interpretation of the diagram.

Both of the study investigators are certified as independent practitioners in emergency ultrasound by the Canadian Emergency Ultrasound Society. One of the investigators (SS) is a course director of national emergency ultrasound course. The study investigators have been further educated in the technique of elective carotid ultrasound by watching over $6 \mathrm{~h}$ of carotid ultrasound lectures. One of the investigators (SS) reviewed a number of elective carotid ultrasounds not used in the study with the investigator who performed the data abstraction (TB) for training purposes.

\section{Key outcome measures}

The primary outcome was the number and percentage of cases with stenoses greater than $50 \%$ based on Doppler who had stenoses of less than $50 \%$ on B-mode alone. 

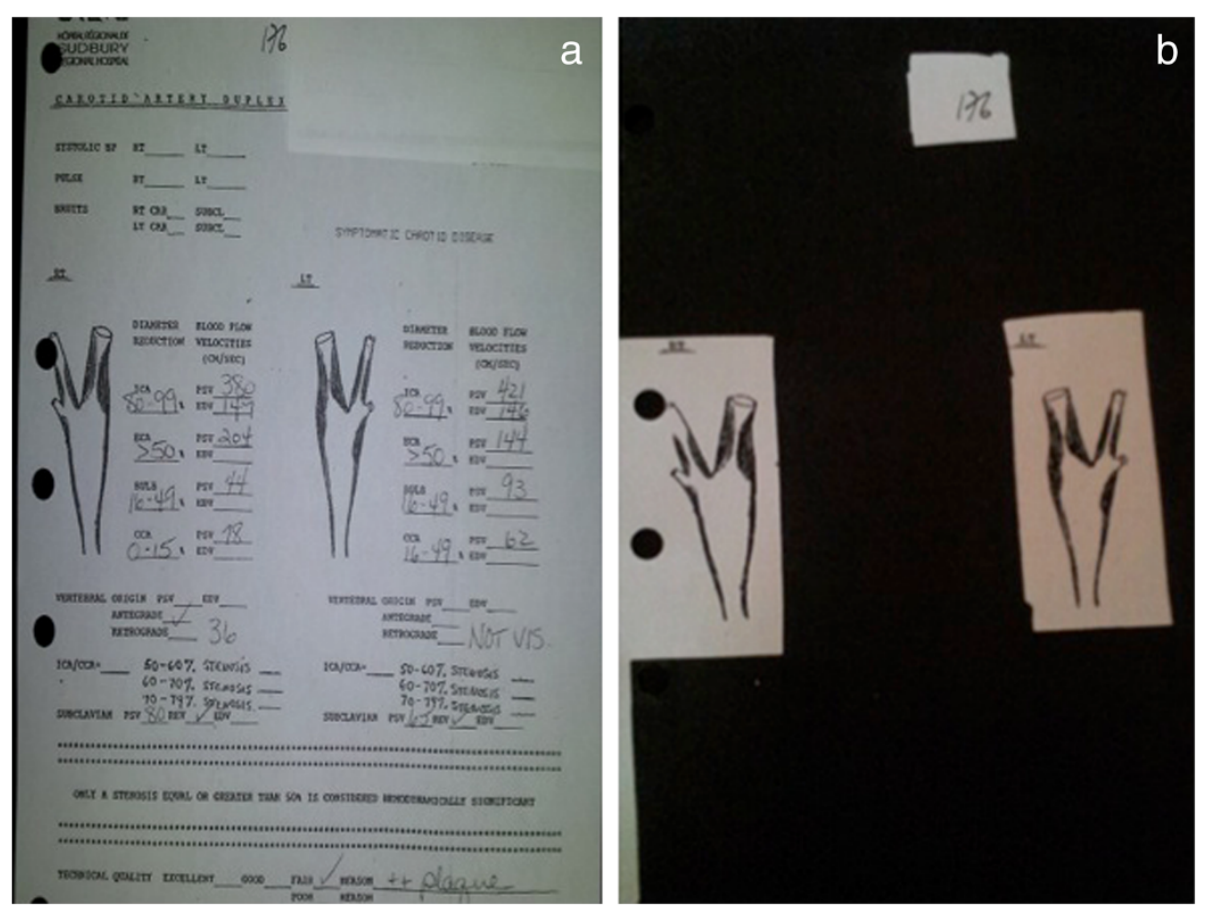

Figure 1 An elective carotid ultrasound report with personal identifiers covered. (a) A photocopy of the same ultrasound report with only the unique identifier number and (b) technologist's drawing visible.

\section{Data management and statistical analysis}

Data were collected and entered into spreadsheets using Microsoft Excel (Microsoft Corporation). The number and percentage of cases with significant stenoses based on Doppler who had non-significant stenoses of less than $50 \%$ on B-mode alone was determined. The sensitivity, specificity, negative predictive value, and positive predictive value were calculated for each group.

\section{Results}

A total of 200 patients were sampled. There were 100 patients from group a and 100 patients from group b. This resulted in 400 carotid ultrasounds: 200 on the left and 200 on the right. The patient's baseline characteristics can be found in Table 1.

The results from the CEA group (group a) are listed in Table 2. The interpretations by our study investigator as compared with the elective ultrasound results yielded a

Table 1 Baseline characteristics

\begin{tabular}{lll}
\hline & Males & Females \\
\hline CEA (group a) & & \\
Number enrolled & 69 & 31 \\
Age range (mean) & $49-87(65.4)$ & $46-84(70.9)$ \\
Stroke Clinic (group b) & & 58 \\
Number enrolled & 42 & $34-88$ (66.9) \\
Age range (mean) & $37-87(64.3)$ & \\
\hline
\end{tabular}

sensitivity of $99.3 \%$, a specificity of $40 \%$, a negative predictive value of $94.7 \%$, and a positive predictive value of $85 \%$.

The results from the stroke clinic group (group b) are listed in Table 3. The interpretations by our study investigator as compared with the elective ultrasound results yielded a sensitivity of $100 \%$, a specificity of $65 \%$, a negative predictive value of $100 \%$, and a positive predictive value of $36.3 \%$. Inter-rater reliability was $65 \%$ for both groups combined. The kappa statistic was 0.61 (95\% CI: $0.38,0.83$ ) for the left carotid and 0.33 (95\% CI: 0.13, 0.53) for the right carotid artery.

When the data for the CEA group (group a) were unblinded and compared to the sonographer's report, it was noted that there were two reports which indicated a greater than $50 \%$ stenosis based on velocity criteria, but no drawing was made on the diagram. In both cases, the radiologist report indicated a stenosis of less than $50 \%$. These two cases were removed from the study. One

\section{Table 2 CEA results (group a)}

\begin{tabular}{lllll}
\hline & & \multicolumn{2}{l}{ Elective ultrasound result } \\
\cline { 3 - 4 } & & $\begin{array}{l}\text { Positive } \\
(\geq 50 \%)\end{array}$ & $\begin{array}{l}\text { Negative } \\
(<50 \%)\end{array}$ & \\
\hline Result of & Positive $(\geq 50 \%)$ & 152 & 27 & 179 \\
reinterpretation & Negative $(<50 \%)$ & 1 & 18 & 19 \\
& & 153 & 45 & 198 \\
\cline { 2 - 4 } & & &
\end{tabular}


Table 3 Stroke clinic results (group b)

\begin{tabular}{lllll}
\hline & & \multicolumn{2}{l}{ Elective ultrasound result } & \\
\cline { 3 - 4 } & & Positive $(\geq \mathbf{5 0 \% )}$ & $\begin{array}{l}\text { Negative } \\
(<\mathbf{5 0 \% )}\end{array}$ & \\
\hline Result of & Positive $(\geq 50 \%)$ & 32 & 56 & 88 \\
reinterpretation & Negative $(<50 \%)$ & 0 & 104 & 104 \\
& & 32 & 160 & 192 \\
\hline
\end{tabular}

ultrasound report documented a greater than $50 \%$ stenosis but only minimal stenosis was drawn by the technologist. The radiologist report also concluded that the stenosis was greater than $50 \%$. This ultrasound scan was kept in the study and accounts for the one false negative found in Table 2.

When the data for the stroke clinic group (group b) were unblinded and compared to the sonographer's report, it was noted that there were seven ultrasound reports in the stroke clinic group which indicated greater than 50\% stenosis in at least one vessel based on velocity criteria, yet no drawing was made on the diagram. These results were compared with the radiologist's interpretation. In six of these cases, the radiologist interpretation concluded that there was less than $50 \%$ stenosis. The radiologist interpreted the remaining case as showing greater than $50 \%$ stenosis. Given the absence of a drawing by the technologist, these seven reports were removed from the study. An eighth report was also removed because both the technologist and the radiologist commented that the study was of poor quality and the results were inconclusive.

Of the 200 patients in the study, two patients were found in both groups. These patients were initially seen in the stroke clinic and referred for a CEA based on their carotid ultrasound results. These scans were unknowingly reviewed twice and thus were left in the study in both groups.

\section{Discussion}

The objective of this study was to determine the frequency with which carotid stenoses of greater than 50\% are found based on velocity criteria when the B-mode ultrasound suggested that the scan was normal or indicative of a stenosis of less than $50 \%$.

In the CEA group, there were 153 ultrasounds which showed a stenosis of greater than $50 \%$ based on velocity criteria. In only one of these cases did the technologist's drawing appear to show a stenosis of less than $50 \%$. The finding of a less than $50 \%$ stenosis on B-mode when the measured velocities indicate a greater than $50 \%$ stenosis is therefore a rare event. This suggests that the use of B-mode alone may detect the vast majority of clinically important carotid stenoses.

In the stroke clinic group, the reinterpretation of the B-mode portion of the scan yielded a sensitivity and negative predictive value of $100 \%$ when compared to velocity criteria for stenoses above or below $50 \%$. The specificity was $65 \%$. The high sensitivity and negative predictive value supports the theory that a B-mode-only scan could be used to screen for significant carotid stenosis. The modest specificity would be acceptable for a screening test.

This pilot study is the first known study of its kind. The results support the theory that it may be possible to use carotid B-mode ultrasound without Doppler to reliably determine if there is stenosis above or below $50 \%$. If further research corroborates and further develops this theory, more ubiquitous screening may be possible. An ultrasound performed with B-mode alone would be simpler and faster to perform. In the authors' experience, a B-mode scan requires less than 5 min to complete when scanning the left and right carotid systems. This would open up the possibility that clinicians who perform POCUS could add carotid stenosis screening to their practice. In fact, the ability of internists trained to perform carotid ultrasound has been studied and yielded positive results. Ray et al. have shown that internists can achieve acceptable sensitivity and high specificity for carotid plaque detection, resulting in high positive and negative predictive values [10].

\section{Limitations}

There are several limitations to this study. The drawing of the sonographer was reinterpreted by one of the study investigators to determine the degree of carotid stenosis using B-mode alone. Due to the subjective nature of the drawing, what the sonographer interpreted as $<50 \%$ stenosis on the diagram may not have been interpreted as such by our investigators. Conversely, what the sonographer interpreted as $\geq 50 \%$ may not have been interpreted as such by our investigators, as was the case for the one false negative in Table 3. Further research should determine the optimal residual diameter or area of residual lumen as seen on B-mode which would allow one to objectively rule out clinically important stenosis.

When developing the study hypothesis, the authors considered using the B-mode images to interpret the degree of stenosis. Unfortunately, the degree of stenosis is determined with elective ultrasound using velocity criteria alone, as measured by the technologist. The B-mode images are not generally used for determining the degree of stenosis. The result is that the images are of insufficient quality to allow for reinterpretation of stenosis. The ideal methodology would have been to prospectively perform the ultrasound with a focus on determining the degree of stenosis using only B-mode, without relying on the velocity criteria. The retrospective nature of this study precluded doing so. Future research should include 
a prospective study comparing an assessment of stenosis using B-mode only to elective ultrasound using velocity criteria.

Our interpretations were dependent on the sonographer's drawing of stenosis on the carotid diagram. In some cases, the drawings may not have reflected the image seen on the screen. There were two cases in group a and seven cases in group b where the technologist failed to draw the stenosis. These cases were withdrawn from the study. This may have affected test characteristics.

Based on the kappa statistic, agreement was substantial for the left carotid artery but only fair on the right. This may be related to the limited experience in the technique of the author performing all of the measurements as well as the subjectiveness of reinterpreting the technologists' drawings.

Although stroke clinic patients were included in the study (group b) to add cases of mild or no stenosis, the rate of significant stenosis was higher than it would have been in an unselected population. This may have resulted in work-up bias. Future research should be performed in populations with a lower rate of stenoses over $50 \%$.

Carotid ultrasound represents only one aspect of the management of carotid stenosis. The results of this study and the theoretical benefit of a B-mode-only carotid ultrasound should be considered in the larger context of the target population most likely to benefit from early detection and evolving research in the medical and surgical management of carotid stenosis.

\section{Conclusions}

This pilot study supports the theory that it may be possible to use carotid B-mode ultrasound without Doppler to reliably determine if there is stenosis above or below $50 \%$. Further research is required before carotid ultrasound using B-mode alone can be recommended.

\footnotetext{
Abbreviations

CS: Carotid stenosis; CEA: Carotid endarterectomy; CVA: Cerebrovascular accident; CCA: Common carotid artery; ICA: Internal carotid artery;

ECA: External carotid artery; POCUS: Point-of-care ultrasound.
}

\section{Competing interests}

The authors declare that they have no competing interests.

\section{Authors'contributions}

SS and TB made substantial contributions to the design, analysis and interpretation of data, the drafting and revision of the manuscript. SS conceived of the study. TB acquired the data. Both SS and TB gave their final approval and agreed to be accountable for all aspects of the work.

\section{Acknowledgements}

The authors wish to thank Dr. Ray Wiss for his invaluable review of the manuscript as well as Vic Sahai and Larry Stitt for their valuable statistical assistance.

\section{Author details}

${ }^{1}$ Emergency Department, Sunnybrook Health Sciences Centre, 2075 Bayview Avenue, Toronto, ON M4N 3M5, Canada. ${ }^{2}$ Emergency Department, Health Sciences North, 41 Ramsey Lake Road, Sudbury, ON P3E 5J1, Canada.
Received: 20 June 2014 Accepted: 9 September 2014

Published online: 01 October 2014

\section{References}

1. Acerra JR, Iskyan K, Qureshi ZA, Sharma RK (2009) Rebuilding the health care system in Afghanistan: an overview of primary care and emergency services. Int J Emerg Med 2(2):77-82

2. Feigin VL, Lawes CMM, Bennett DA, Barker-Collo SL, Parag V (2009) Worldwide stroke incidence and early case fatality reported in 56 population-based studies: a systematic review. Lancet Neurol 8:355-369

3. North American Symptomatic Carotid Endarterectomy Trial Collaborators (1991) Beneficial effect of carotid endarterectomy in symptomatic patients with high-grade stenosis. N Engl J Med 325:445-453

4. Rahimzai M, Amiri M, Burhani NH, Leatherman S, Hiltebeitel S, Rahmanzai AJ (2013) Afghanistan's national strategy for improving quality in health care. Int J Qual Health Care 25(3):270-276

5. Franco LM, Marquez L (2011) Effectiveness of collaborative improvement: evidence from 27 applications in 12 less developed and middle-income countries. BMJ Qual Saf 20:658-665

6. Lavenson GS Jr (2005) The American Vascular Association Screening Program: rationale, method, guidelines and progress. J Vasc Ultrasound 29:85-89

7. Wolff T, Guirguis-Blake J, Miller T, Gillespie M, Harris R (2007) Screening for asymptomatic carotid artery stenosis. Evidence synthesis No. 50. AHRQ publication No. 08-05102-EF-1. Agency for Healthcare Research and Quality, Rockville

8. Campbell J, Buchan J, Cometto G, David B, Dussault G, Fogstad H, Fronteira I, Lozano R, Nyonator F, Pablos-Mendez A, Quian EE, Starrs A, Tangcharoensathien $V$ (2013) Human resources for health and universal health coverage: fostering equity and effective coverage. Bull World Health Organ 91:853-863

9. Ratchford EV, Jin Z, Di Tullio MR, Salameh MJ, Homma S, Gan R, Boden-Albala B, Sacco RL, Rundek T (2009) Carotid bruit for detection of hemodynamically significant carotid stenosis: the Northern Manhattan study. Neurol Res 31:748-752

10. Ray A, Tamsma JT, Hovens MMC, Roodt J, Huisman MV (2010) Accuracy of carotid plaque detection and intima-media thickness measurement with ultrasonography in routine clinical practice. Eur J Intern Med 21:35-39

doi:10.1186/s13089-014-0017-x

Cite this article as: Bhandari and Socransky: Is B-mode ultrasound alone a sufficient screening tool for carotid stenosis? A pilot study. Critical Ultrasound Journal 2014 6:17.

\section{Submit your manuscript to a SpringerOpen ${ }^{\circ}$ journal and benefit from:}

- Convenient online submission

- Rigorous peer review

- Immediate publication on acceptance

- Open access: articles freely available online

- High visibility within the field

- Retaining the copyright to your article

Submit your next manuscript at $>$ springeropen.com 